\title{
Acute Epidural Hematoma Compressing the Dominant Sigmoid Sinus as an Unusual Cause of Intracranial Hypertension: Case Report and Review of Literature
}

\author{
Gustavo Rajz1, Ido Ben Zvi1 ${ }^{*}$, José E. Cohen², Shalom Michowiz' \\ ${ }^{1}$ Department of Pediatric Neurosurgery, Schneider Children's Medical Center of Israel, Petach Tikvah, Israel \\ ${ }^{2}$ Department of Neurosurgery, Hadassah-Hebrew University Medical Center, Jerusalem, Israel \\ Email: ${ }^{*}$ idobenz@gmail.com
}

Received 25 January 2014; revised 25 February 2014; accepted 3 March 2014

Copyright (C) 2014 by authors and Scientific Research Publishing Inc.

This work is licensed under the Creative Commons Attribution International License (CC BY).

http://creativecommons.org/licenses/by/4.0/

(c) (i) Open Access

\begin{abstract}
Post traumatic dural sinus vein stenosis has been rarely described in pediatric population. We present a case of a 9-year-old child that had sustained a head injury after a fall from height causing an acute epidural hematoma compressing the dominant sigmoid sinus. The patient had developed sub acutely signs and symptoms of increased intracranial pressure. Prophylactic treatment with anticoagulants was initiated despite the presence of an intracranial bleeding. Clinical and radiological improvements were achieved. We had also reviewed the literature regarding this uncommon entity and discussed other existing diagnostic and therapeutic alternatives. Further gathering of information is essential in order to form a therapeutic protocol.
\end{abstract}

Keywords

Dural Sinus Stenosis, Epidural Hematoma, Intracranial Hypertension

\section{Introduction}

Posttraumatic sinodural vein stenosis has been rarely described in pediatric population. Most of the reported cases are associated with depressed fractures and tend to present a good clinical evolution without specific treatment. However, development of increased intracranial hypertension due to venous compromise is among the most feared complications.

*Corresponding author.

How to cite this paper: Rajz, G., et al. (2014) Acute Epidural Hematoma Compressing the Dominant Sigmoid Sinus as an Unusual Cause of Intracranial Hypertension: Case Report and Review of Literature. Open Journal of Modern Neurosurgery, 4, 76-80. http://dx.doi.org/10.4236/ojmn.2014.42016 
To the best of our knowledge acute epidural hematoma compressing the dominant sigmoid sinus and progressively leading to intracranial hypertension was not previously reported. We present a case of a traumatic posterior fossa epidural hematoma, causing compression of the dominant sigmoid sinus that consequently led to signs and symptoms of intracranial hypertension. We will also review the literature and discuss the therapeutic implications of this association.

\section{Case Description}

A 9-year-old previously healthy male child sustained a minor head injury. A day before admittance, while playing on a tree, fell down approximately 3 meters. No loss of consciousness or vomiting was reported. He was taken to the pediatric emergency room and was released after a thorough neurological exam. The day after, his father noticed that the boy was sleepy and after he vomited a couple of times, the parents decided to bring him to the hospital again.

\subsection{Examination}

On arrival to the pediatric emergency room the child was fully conscious, vital signs were normal. He reported a strong headache and suffered from photophobia. On physical examination a number of bruises were demonstrated and no neurological deficits were noticed.

\subsection{Imaging}

A plain computerized tomography (CT) scan was performed that demonstrated a right sided epidural hematoma, posterior to the Mastoid bone in the groove of the sigmoid sinus. After consulting with the radiology attending physician a venous computerized tomography (CTV) was performed to rule out sinus vein thrombosis (SVT). On CTV, SVT was in fact ruled out, but it appeared that the epidural hematoma had severely compressed the sigmoid sinus (Figure 1).

\subsection{Management}

The child was admitted to the pediatric surgery ward for observation and monitoring. Two days after his admittance, the child complained of blurred vision. On physical examination a deficit of the sixth nerve, initially on the right and then in both eyes was demonstrated. It later progressed to a complete bilateral $6^{\text {th }}$ nerve palsy. An eye exam demonstrated papilledema on the nasal side of both eyes. A treatment with Acetazolamide was then initiated and another CTV scan was performed that did not demonstrate a worsening in the initial findings.

Due to the worsening of his clinical condition, the child was transferred to the pediatric intensive care unit (PICU). A multi-disciplinary meeting was called in because of the dilemma of whether or not to prevent the thrombus formation by using anti-coagulants or wait because of the presence of the acute bleeding. It was de-

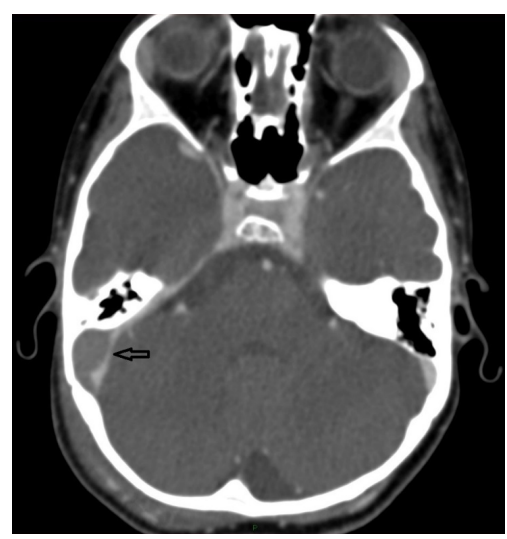

(a)

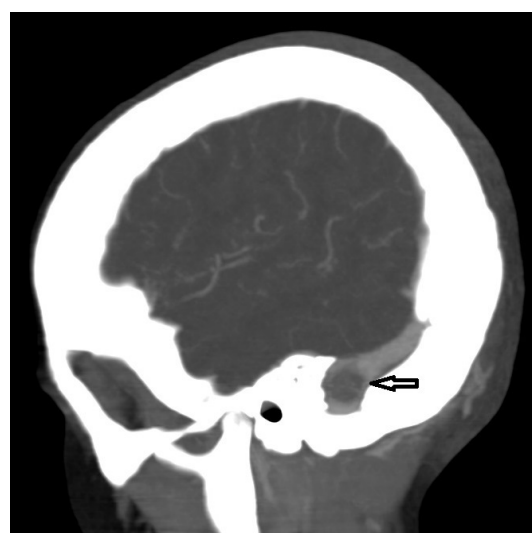

(b)

Figure 1. CTV (a) axial and (b) sagittal views. The thinning of the sigmoid sinus is marked by the arrow. Blood flow is still apparent. 
cided to initiate a treatment with Heparin establishing a PTT target level of 1.5 times the basic level (normal values are 24 - 40).

A follow up venous magnetic resonance imaging (MRI-MRV) was performed demonstrating a full stenosis of the dominant sigmoid sinus (Figure 2(a)).

Despite full stenosis no modifications of treatment were made because of the child's clinical condition that remained unchanged. After another three days of careful observation, clinical improvement was noticed, with progressive improvement of eye movement and a subjective improvement in headaches.

Because of the child's improvement, treatment with Heparin was substituted with low molecular weight Heparin (LMWH) using a prophylactic dosage. After one more day the child was moved back to the surgical ward. A couple of days later a follow up MRI-MRV scan was performed that demonstrated a renewed perfusion of the previously stenosed sigmoid sinus (Figure 2(b)).

After improvement, both clinical and by imaging and follow up eye exam, the child was released from the hospital with LMWH for four more days. In a follow up checkup in our pediatric neurosurgical clinic the child had a healthy appearance, with no headache or other complaints, and with no apparent diplopia and a normal eye examination with a resolution of the papilledema.

\section{Discussion}

Posttraumatic intracranial hypertension secondary to depressed midline or retromastoid skull fracture compressing the superior sagittal sinus or the dominant sigmoid sinus respectively has been scarcely described [1]-[3] with no description in the pediatric population. In these cases symptomatic intracranial hypertension develops at a subacute stage, frequently after a patient was already discharged. Patients usually present with headaches, cranial nerve alterations or encephalopathy. The patient discussed herein, presented a small acute epidural hematoma, strategically located over the dominant sigmoid sinus. The effect of the hematoma over the sigmoid sinus was already detected at the presentation with uncontrasted and contrasted CT. Upon development of symptoms of elevated ICP additional imaging methods were performed as described above. Conservative treatment was initiated with benefit both clinical and radiological. Even though the hematoma was not considered surgical based on clinical and radiological criteria, compression of a dominant sigmoid sinus by an epidural hematoma was not previously reported. Large and medium size epidural hematomas are usually managed surgically, however conservative treatment is frequently chosen in management of small or less accessible hemorrhages such as the one described above.

In our case, a direct pressure caused by the epidural hematoma induced the stenosis of the sigmoid sinus thus impeding the venous return and elevating the ICP as demonstrated by the sixth nerve palsy, papilledema and headaches.

There is little consistency in literature in regards to the best imaging means to use in order to demonstrate si-

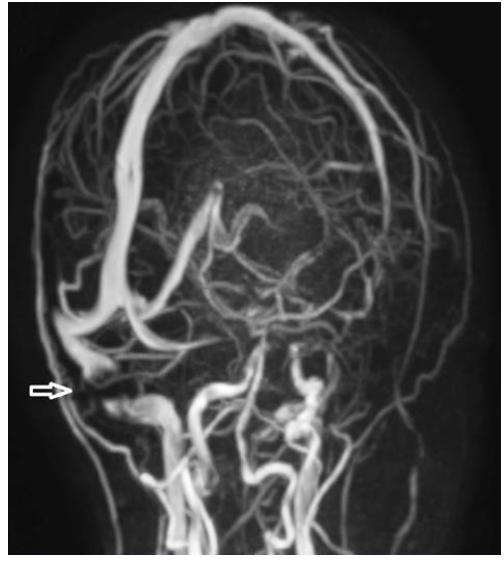

(a)

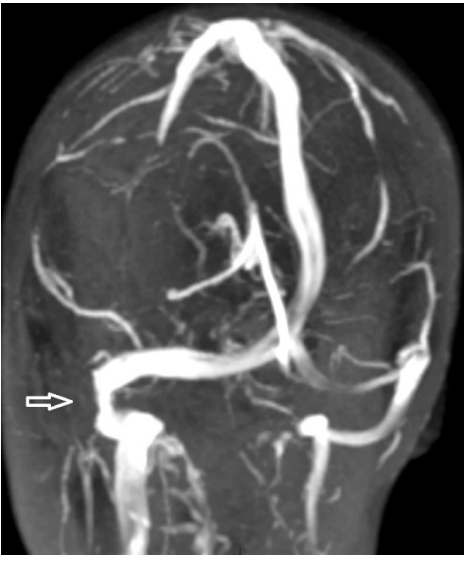

(b)

Figure 2. (a) An MRV left oblique-sagittal view. The arrow marks the stenosed sigmoid sinus. (b) An MRV right obliquesagittal view. The arrow marks the improved flow of the sigmoid sinus. 
nus vein stenosis/thrombosis. In a review of literature of SVT from 2009 [4] they concur that MRI-MRV is the most sensitive imaging test although they also state the importance of specialized personnel in order to rule out anatomical variants that simulate thrombosis such as a non-dominant sinus that can create a false positive result. In that review they consider digital subtraction angiography (DSA) as a complimentary test. That is probably due to the fact that DSA is an invasive test, even though it has a higher ability to distinguish sinus stenosis from thrombosis [5]. In a French group's double case study of sinus stenosis [2] they suggest a direct retrograde cerebral venography and venous pressure readings that can aid diagnosis and follow up by demonstrating an upstream and downstream pressure gradient. In our case a CTV and an MRI-MRV done one day after the other demonstrated a non-complete stenosis with the first test and a complete stenosis with the second test, despite the fact that the child's symptoms did not worsen from the time of the first test to the time of the second test. We therefore theorize a better reliability of the CTV in diagnosing sinus vein stenosis when compared with MRIMRV. We of course cannot rule out a radiological deterioration even when no clinical change was noticed.

There are several possibilities of treatment described in literature. Conservative treatment with the use of prophylactic anticoagulation therapy and supportive care [3]. In a review published in 2013 by Pikis et al. of 18 cases of SVT collected over a period of 22 years they conclude that anticoagulant therapy should be reserved for severe or deteriorating patients or for patients with concomitant prothrombotic disorders [6].

Stenting of the occluded vessel to allow its expansion and maintain flow [7] is another alternative that has been described. Surgery has been offered as a treatment in case of failure of a conservative approach [3]. In some cases surgery had been offered as the first line of treatment because of the low probability of success with a non-surgical remedy [1]. The last quote was taken from a case report in which a depressed fracture had caused the occlusion which can explain better the surgical approach as a first line of treatment. To note that in one case report the author had performed several lumbar punctures as a means to lower the ICP without further intervention [8].

Because information about sinus vein stenosis is relatively scarce it is easier to demonstrate information regarding SVT. In the past, mortality due to SVT reached numbers as high as 30\% - 50\% [4]. The modern imaging era and the therapeutic options of present day had decreased these numbers to $7 \%-13.6 \%$ [9]. These percentages differ in accordance with the symptomatology. Patients who presented to the hospital with isolated intracranial hypertension syndrome had better outcome than patients with other presenting factors or symptoms such as older age, coma, seizures, altered mental status etc.

A meta-analysis done by Dentali et al. [10] had demonstrated a recurrence rate of $2.8 \%$. As for stenosis the data consists of a handful of articles, most of whom are case reports. In an American-Chinese article comparing 34 cases of SVT with 34 cases of sinus vein stenosis a percentage of mortality regarding SVT was reported as $4.3 \%$ in the acute phase, that is within 7 days [5] and 3.4\% more die within 30 days. As to sinus vein stenosis, they state that delayed treatment could cause serious consequences such as optic atrophy and blindness, but they do not address mortality in these cases. Moreover they state that sinus stenosis has a high hospital admission rate costing up to 444 million dollars per year in the US. In a Japanese case report and review cited earlier [3] other 5 case reports were cited, all of whom were treated in one of the ways we stated before, $4 / 5$ of whom reported good recovery and 1 reported moderate disability.

\section{Conclusion}

This case report is exceptionally rare as the rest of the reports (already a few cases worldwide) regarding traumatic sinus stenosis are due to a depressed fracture and the subsequent occlusion of the sinus, whereas in our case, the stenosis is due to an epidural hematoma, making the therapeutic decision in our case much more challenging. Any use of anti-coagulant medication has to be thought of carefully in the set-up of acute bleeding. Because of that and because of a minority in similar case reports published, no clear guidelines can be given at this point as to what is the right treatment for these patients. It has been our impression that CTV imaging is more sensitive than MRV not only for detecting sinus vein stenosis but also for describing more accurately how stenosed the vessel really is.

\section{Disclosure}

The authors report no conflict of interest concerning the findings specified in this paper. 


\section{References}

[1] Donovan, D.J. (2005) Simple Direct Skull Fracture Causing Sagittal Sinus Stenosis and Increased Intracranial Pressure: Case Report and Review of the Literature. Surgical Neurology, 63, 380-384. http://dx.doi.org/10.1016/j.surneu.2004.06.020

[2] Fuentes, S., Metellus, P., Levrier, O., Adetchessi, T., Dufour, H. and Grisoli, F. (2005) Depressed Skull Fracture Overlying the Superior Sagittal Sinus Causing Benign Intracranial Hypertension. Description of Two Cases and Review of the Literature. British Journal of Neurosurgery, 19, 438-442. http://dx.doi.org/10.1080/02688690500390193

[3] Yokota, H., Eguchi, T., Nobayashi, M., Nishioka, T., Nishimura, F. and Nikaido, Y. (2006) Persistent Intracranial Hypertension Caused by Superior Sagittal Sinus Stenosis Following Depressed Skull Fracture. Journal of Neurosurgery, 104, 849-852. http://dx.doi.org/10.3171/jns.2006.104.5.849

[4] Filippidis, A., Kapsalaki, E., Patramani, G. and Fountas, K.N. (2009) Cerebral Venous Sinus Thrombosis: Review of the Demographics, Pathophysiology, Current Diagnosis and Treatment. Neurosurgical Focus, 27, E3. http://dx.doi.org/10.3171/2009.8.FOCUS09167

[5] Meng, R., Dornbos, D., Meng, L., Wu, Y., Liu, Y., Li, G., Li, G., Li, S., Sun, F., Wang, X., Ding, Y. and Ji, X. (2012) Clinical Differences between Acute CVST and Non-Thrombotic CVSS. Clinical Neurology and Neurosurgery, 114, 1257-1262. http://dx.doi.org/10.1016/j.clineuro.2012.03.036

[6] Pikis, S., Moscovici, S., Itshayek, E. and Cohen, J.E. (2013) Cerebral Sinodural Thrombosis Following Minor Head Injury in Children. Journal of Clinical Neuroscience, 20, 481-484. http://dx.doi.org/10.1016/j.jocn.2012.09.009

[7] Arac, A., Lee, M. and Steinberg, G.K. (2009) Efficacy of Endovascular Stenting in Dural Venous Sinus Stenosis for the Treatment of Idiopathic Intracranial Hypertention. Neurosurgical Focus, 27, 1-8.

[8] Van Den Brink, W., Pieterman, H. and Avezaat, C.J. (1996) Sagittal Sinus Occlusion, Caused by an Overlying Depressed Cranial Fracture, Presenting with Late Signs and Symptoms of Intracranial Hypertension: Case Report. Neurosurgery, 38, 1044-1046. http://dx.doi.org/10.1097/00006123-199605000-00039

[9] Ferro, J.M., Canhao, P., Stam, J., Bousser, M.G. and Barinagarrementeria, F. (2004) Prognosis of Cerebral Vein and Dural Sinus Thrombosis. Results of the International Study on Cerebral Vein and Dural Sinus Thrombosis (ISCVT). Stroke, 35, 664-670. http://dx.doi.org/10.1161/01.STR.0000117571.76197.26

[10] Dentali, F., Gianni, M., Crowther, M.A. and Ageno, W. (2006) Natural History of Cerebral Vein Thrombosis. Blood, 108, 1129-1134. 